\title{
Reflections on Stephan Mueller's talents
}

\section{by Karl Fuchs}

\author{
Dear Doris, \\ Dear participants of the $\mathbf{1}^{\text {st }}$ Stephan Mueller Conference,
}

I have been asked to contribute with some informal personal impressions from the early time in Stephan Mueller's scientific career at Karlsruhe and before to the opening of this first Stephan Mueller conference. These times between 19591972 are unforgettable because I met a colleague, an advisor and, last not least, a friend. What did we learn from Stephan almost 40 years ago? What should we keep in mind as we try to master the future of Earth Sciences in Europe?

Out of many advises Stephan had a simple rule which I want to share with you. It may surprise you, he himself followed it almost $100 \%$, and he transmitted it to his students and colleagues at Karlsruhe in the early sixties: A good presentation has always to start with a seismogram. I will follow this rule now:

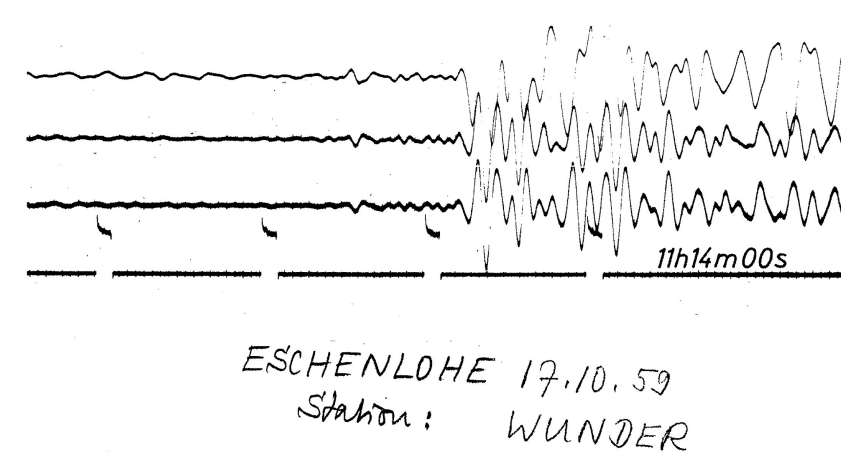

Fig. 1. Eschenlohe Seismogram 1959.

1959, the beginning of the large DSS program to explore the crust and upper mantle in central Europe. And Stephan was in charge of the main data center for paper records from all over Germany in Stuttgart.

Eschenlohe, the strongest shot point north of the Alps (10 to instantaneous quarry blast; unbelievable today). The recording instruments still analogue, a complete mixture of all possible types of instruments, as if out of the German $\mathrm{Mu}$ seum in Munich. Weak $\mathrm{P}_{\mathrm{n}}$ forerunner and strong PmP supercritical reflection, at that time just barely recognized in its importance. A crew of two people was driving for 2-3 days to fetch this single seismogram.

Why do I follow Stephan's advice to start always with a seismogram? - Why I am telling this sentimal story about the Eschenlohe seismogram archived by Stephan in 1959? It took me a while to understand the secret of the success of his recipe: A seismogram or seismogram section represents the complete data set, full of useful information, not just the picked arrival times and without the sophisticated bias of the interpreter expressed in the so called correlations. A model is always an abstraction from the observations and a poor, biased projection of the original data without its rich information content. And - most important: the surprises are in the data, NOT in the model !!

We all know and appreciate Stephan's mastery in quantitative model building integrating various disciplines of seismology and geophysics; and yet, Stephan's research was data driven! What does this mean?

He was an arduous hunter of data (not just an archivar), because he was convinced that new ideas, surprises, the unexpected would only be encountered if we strive to conquer the unknown, the many terra incognitas.

Once at Karlsruhe I remarked to Stephan when he was heading for the next large field experiment that we should also take time for a more detailed analysis of the data which we had been compiling in the institute in a very short time. His answer: "Karl, when we are old we have all the time to analyse the data, now it is time to hunt for them!" New data were Stephan's life elixir! What happened to these new data?

There is a very important aspect of Stephan's scientific talents: he was not only a hunter, but also a master in storing data in his mind, searching for new connections, and testing them, if necessary also by advanced quantitative modelling, but always with the powerful ability of the human mind for mental mapping through an enormous amount of data with incredible speed searching for connections in parallel in multidimensional phase diagrams in his mind.

On some occasions we could get a glimpse of what his mind was up to with the huge amount of fresh and "fossile" 


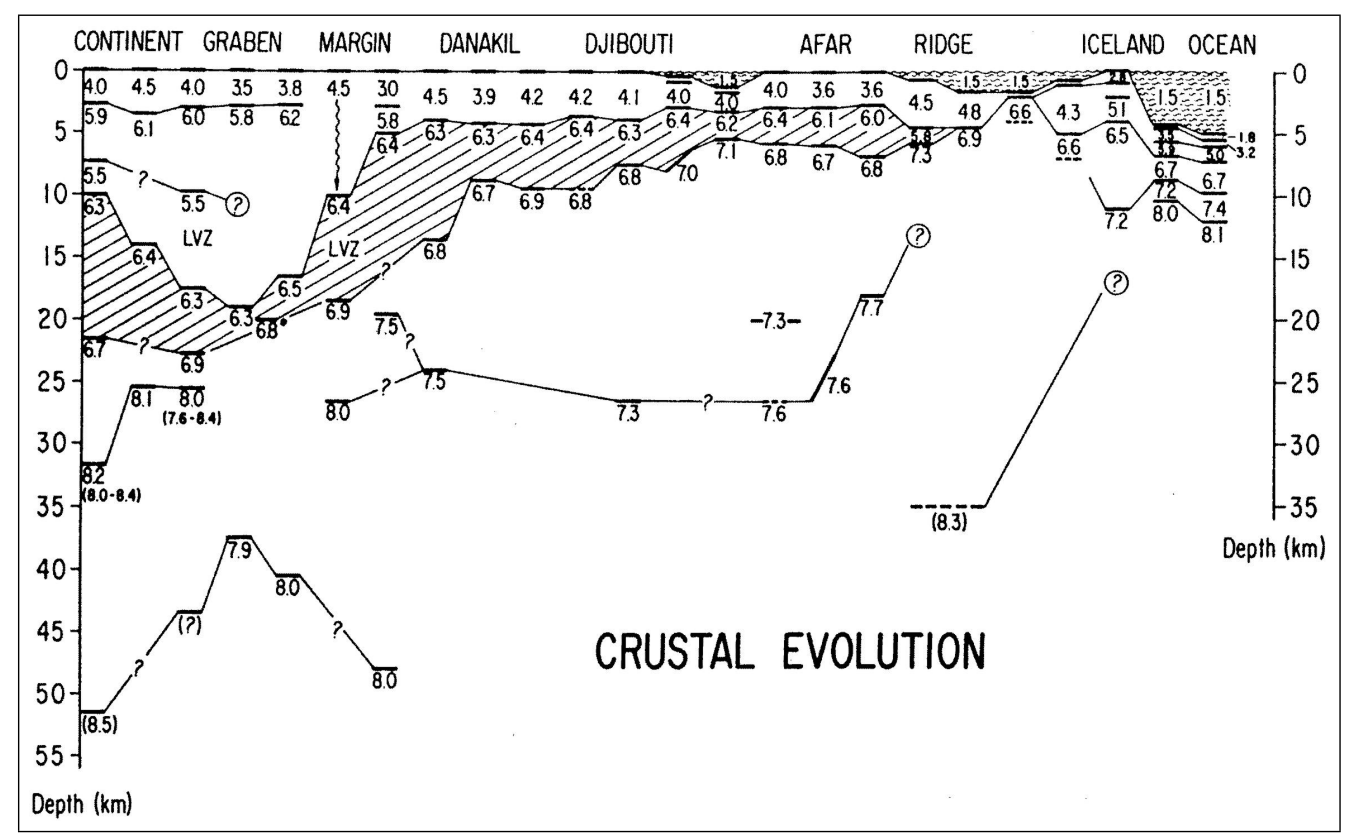

Fig. 2. Stephan's mental map of Crustal Evolution (Mueller, 1978).

Geophys. J. R. astr. Soc. (1966) 10, 525-538.

Geophys. J. R. astr. Soc. (1966) 10, 539-548.

\title{
Seismic Studies of the Earth's Crust in Continents
}

\section{I: Evidence for a Low-Velocity Zone in the Upper Part of the Lithosphere*}

\author{
Stephan Mueller $\dagger$ and Mark Landisman
}

\section{II: Analysis of Wave Propagation in Continents and Adjacent Shelf Areas*}

\author{
Mark Landisman and Stephan Mueller $\dagger$
}

(Received 1965 May 10)

* Southwest Center for Advanced Studies, Geosciences Division, Contribution No. 16. $\dagger$ On leave from Technische Hochschule Karlsruhe, Germany.

Fig. 3. Titles of the two original papers on the LVZ.

information (including that from literature !). At instances you could see him mentally mapping, and to his friends he would allow a glimpse into his attempts to connect information, trying to enter even into the fourth dimension towards a picture of crustal evolution (Fig. 2).
This diagram, first seen during the early 70s, is highly relevant to this Conference. It also leads us to understand that Stephan was reaching from static models into the time dimension. I know how much he was always eager to interact with the best of geologists like Henning Illies at Karlsruhe to 


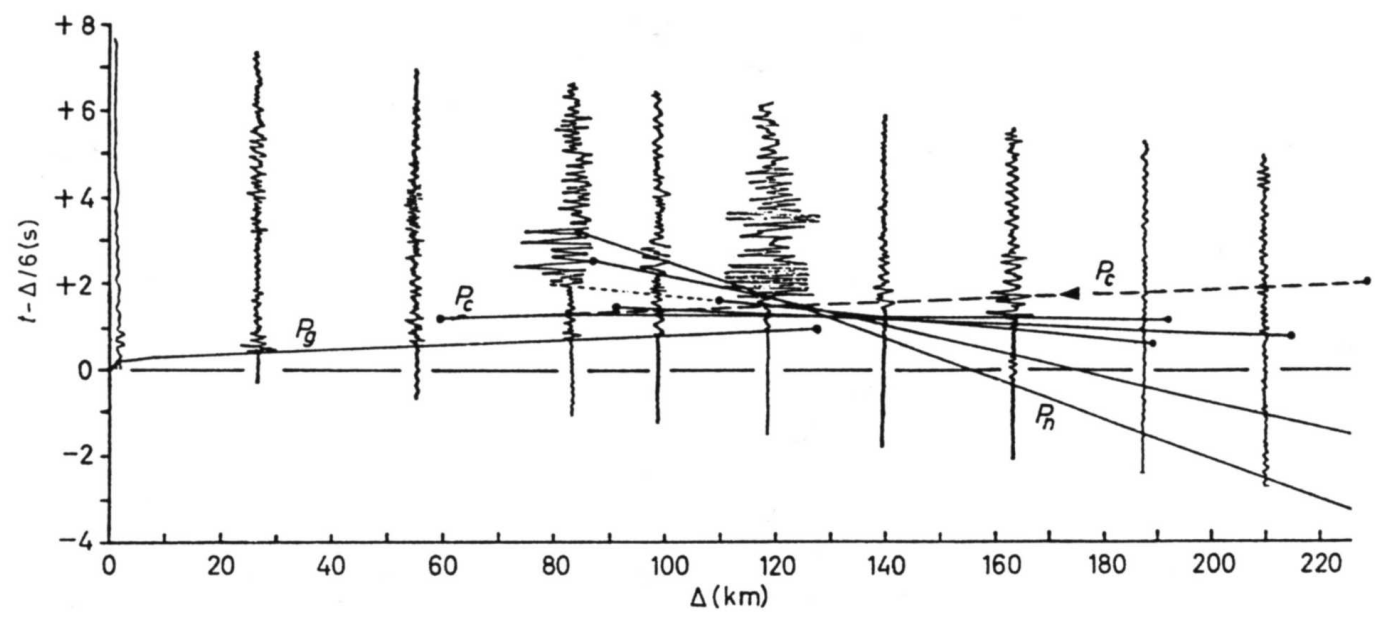

Fig. 4a. Haslach Section with phase Pc characteristic for the LVZ.

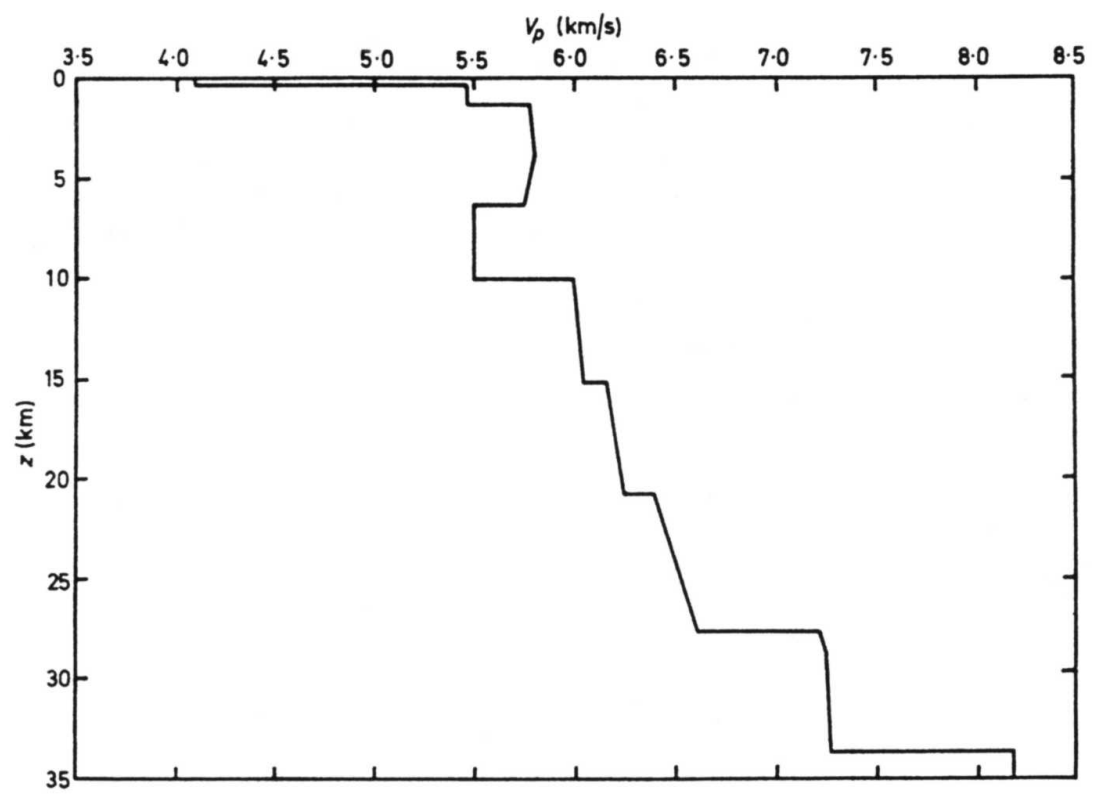

Fig. 4b. Crust with low velocity zone (LVZ) beneath a high velocity lid.

widen static geophysical models as a Geophysicist into the fourth dimension of the Earth.

This is why Stephan was so intrigued and attracted by the new tools like GPS and the new mantle wide tomography. As in seismic exploration, observations are transformed into images of the Earth interior which allow direct access to the full range of information so directly as if you almost plan to drill into such a data set. This offers a new way to search for structure in data allowing mental connections to numerous Earth science data sets without loosing the freshness of the data. There is another side to Stephan's talents. On the left hand side there are three letters L, V, Z standing for low velocity zone; they signify the "sialic low velocity zone". Together with a typical velocity distribution very well known to Stephan's collaborators from Karlsruhe and Zurich and friends around the world.
The discovery of this low velocity zone in the mid 60 s is an excellent example of Stephan's ability to observe, to connect and to discover the unexpected (Fig. 3).

These two papers jointly published by Stephan Mueller and Mark Landisman demonstrate Stephan's mastery to connect widely separated data sets from all over continents as a basis for the test of the hypothesis of a continentwide existence of a low velocity zone in the upper crust.

Please note: It was NOT alone the reinterpretation of a single seismic profile, the famous 1948 Haslach explosion in S.-Germany, NOT a better error estimate, which would have convinced the seismic community: BUT it was the connection with two different seismic data sets: the newly recognised tool of vertical reflection information, and the world wide collection of $\mathrm{Pc}$ arrivals

This still is and has always been a crucial problem, how 


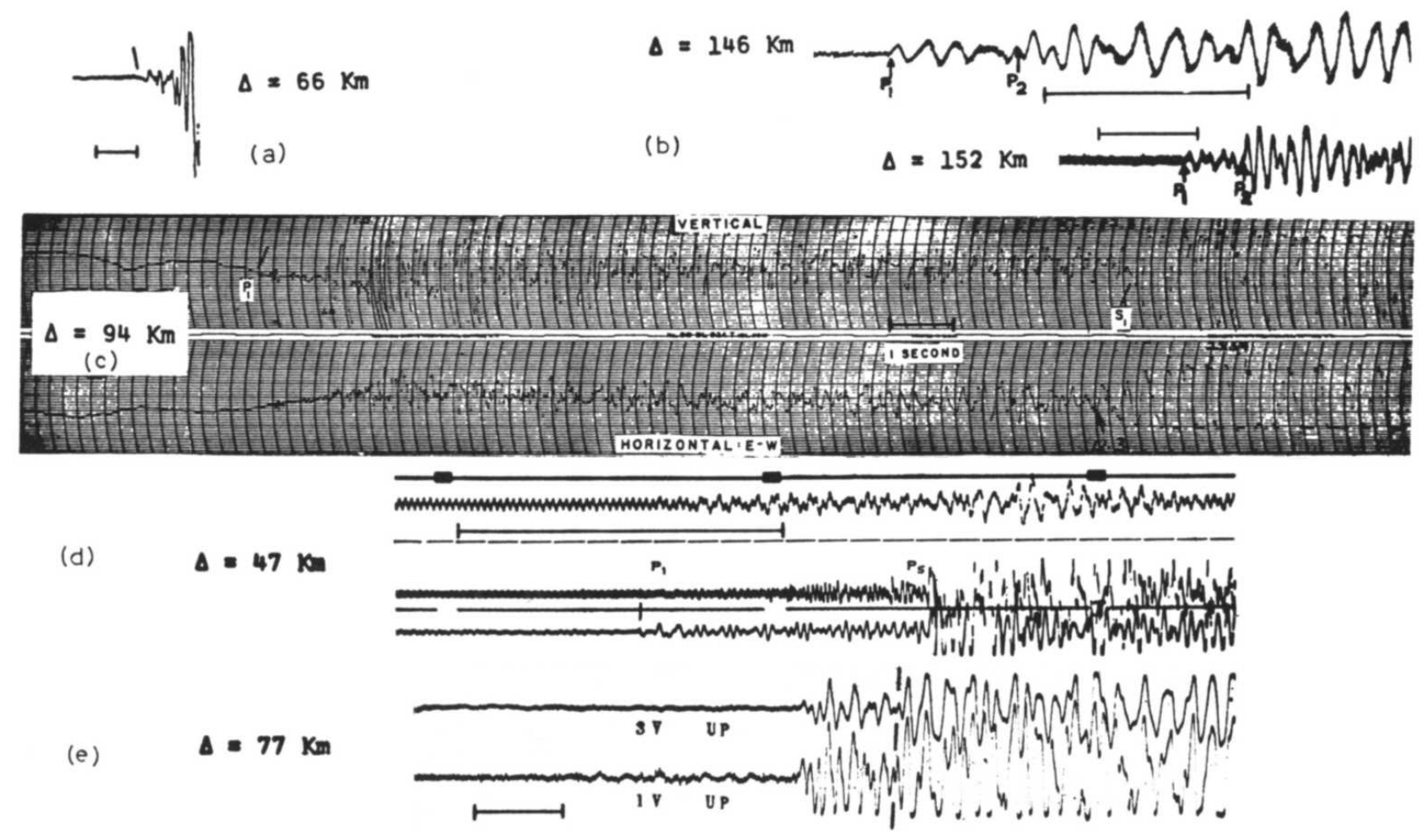

Fig. 5. Pc examples from around the world.

to convince our fellow scientists, the scientific community of the data and of their connections which we have developed in our minds? There is no other way but to spell them out and to communicate them to the community.

Stephan and Mark had chosen a special way which was rather unusual in the seismic community at that time and even today. With our mathematical and physical education we tend to be easily satisfied by a robust model with drastically reduced rms errors obtained by fitting picked to calculated observations. But did we pick the right phases? Did we choose the right class of models?

Stephan let the readers share the relevant data set in his mind which convinced him of the existence of the Pc phase following the first arriving Pg, sometimes with quite strong amplitudes: from Heligoland explosion through Montana, Australia, Baltic Shield and other locations. - In a way, this is what is known as geological reasoning: "Look here and look there! How do you connect these observations? What is the connecting model?"

Two talents are very important in the exploration and understanding of this planet: first, new discoveries originate from the ability to recognize new patterns or surprizing structure in data and unexpected connections expressed as a testable hypothesis; and second, intuition and luck based on broad experience is the most important ingredient in the exploration of this world.

I do not want to become philosophical, Stephan was always very pragmatic. However, I have one concern: the growth of fast, powerful large memory computers provides us with enormous opportunities. We are fascinated by the huge and realistic amount of synthetic or calculated data of processes in complex 3-D- and even 4-D-models.

But, if we are not developing our ability to feed and store observed information into our minds and to operate on them, then we are endangering our ability to discover the unexpected. That could well mean that we are losing track of the roots of our science, because, science is not living by dreams alone (our dreams reflect very much our present knowledge), but by the unexpected, by the surprise which we encounter in new observations and their connections.

This series of Stephan Mueller conferences is a very good chance that we continue in his spirit to master the future of Earth sciences in Europe. Stephan would say: let's do it !

\section{References}

Landisman, M. and Mueller, St.: Seismic studies of the Earth's crust in continents. - II. Analysis of wave propagation in continents and adjacent shelf areas, Geophys. J. R. Astr. Soc., 10, 539-548, 1966.

Mueller, St. and Landisman, M.: Seismic studies of the Earth's crust in continents. - I. Evidence for a Low-velocity zone in the upper part of the lithosphere, Geophys. J. R. Astr. Soc., 10, 525-538, 1966.

Mueller, St.: Evolution of the Earth's Crust, in: Ramberg, I. B. and Neumann, E.-R. (Eds.): Tectonics and Geophysics of Continental Rifts, D. Reeidel Publishing Co., Dordrecht, Holland, 11-12, 1978. 